\title{
CABALLOS, RATONES, GEMAS Y PULGAS: UN DEBATE OLVI- DADO SOBRE EL FUNDAMENTO ÚLTIMO DEL VALOR ECONÓMICO Y DE LOS PRECIOS ENTRE TOMÁS DE AQUINO, CONRADO SUMMENHART, DOMINGO DE SOTO, Y DIEGO DE COVARRUBIAS
}

\author{
HORSES, MICE, GEMS AND FLEAS: FORGOTTEN DEBATE \\ BETWEEN THOMAS AQUINAS, CONRADO SUMMENHART, \\ DOMINGO DE SOTO, AND DIEGO DE COVARRUBIAS ABOUT THE \\ LAST FOUNDATION OF ECONOMIC VALUE AND PRICES
}

\author{
ÁlVARO PERPERE VIÑUALES \\ Pontificia Universidad Católica Argentina
}

\section{RESUMEN}

La amplia mayoría de los trabajos dedicados al fundamento filosófico del valor económico desarrollado por la llamada "Escuela de Salamanca" destacan el rol otorgado a la subjetividad. Sin embargo, la apelación que hacen todos a ejemplos muy similares, y la continua referencia a las ideas de quienes los precedieron, han dado a mi juicio una imagen demasiado homogénea de toda esta tradición, y ha invisibilizado un complejo y sutil debate suscitado dentro suyo. Por ello, en el presente artículo me propongo mostrar que, a pesar de la aparente continuidad conceptual, es posible reconocer un interesante debate entre Conrado Summenhart, Domingo de Soto, y Diego de Covarrubias sobre esta temática de filosofía de la economía. En mi opinión, el rol de la subjetividad ocupa lugares muy diferentes en unos y otros, dando lugar a concepciones diferentes respecto del fundamento último del valor económico. 
Palabras clave: filosofía de la economía, Escuela de Salamanca, teoría del valor, precio justo.

\section{ABSTRACT}

The academic literature that analyses the philosophical foundation of economic value developed by the so-called "School of Salamanca" highlights the role given by this school to subjectivity. In my opinion, however, there is a perception that all of these theologians are part of a homogeneous tradition probably because they appeal to very similar examples and they make continuous references to the ideas of those who preceded them. This situation has made a complex and subtle debate raised within them invisible. Therefore, in this article I intend to show that, despite the apparent conceptual continuity, it is possible to recognise an interesting debate among Conrado Summenhart, Domingo de Soto, and Diego de Covarrubias on this philosophy of economy central question. As I will try to show, the role of subjectivity occupies very different places in each of them, and due to this, it is possible to recognise among them alternatives conceptions regarding the ultimate foundation of economic value.

Keywords: Philosophy of Economics, School of Salamanca, value Theory, Just Price.

\section{INTRODUCCIÓN}

Al analizar el fundamento último de la valoración económica, es decir, aquello por lo cual las personas ponen un precio a los objetos que intercambian en el mundo comercial, la tradición escolástica, y más tarde la llamada "Escuela de Salamanca", apeló, casi unánimemente, a la idea de "estimación" o "estimación común". Diversos historiadores de las ideas económicas han destacado este punto, al que señalaron como un sólido argumento frente a quienes atribuyeron

1 Es de señalar que el concepto mismo de "Escuela de Salamanca" es actualmente discutido por quienes se dedican a este período. El reconocimiento de la vitalidad que se vivía en la vida académica no se limitaba solamente a la Universidad de Salamanca, y la variedad de temas abordados, hace que su exacta delimitación como "Escuela" conlleve algunas dificultades. Este problema está claramente planteado, con abundante bibliografía sobre el tema, en José Barrientos García y María Idoya Zorroza, "Moral y Política en la Escuela de Salamanca. Presentación", Anuario Filosófico, 45 (2012): 242-245. 
a la escolástica una teoría del valor excesivamente objetivista ${ }^{2}$. La reiterada apelación que hicieron los escolásticos a la "estimación común" como fuente de la valoración mostraría que hay en todos ellos una clara conciencia de que toda apreciación económica tiene que estar fundada, al menos en parte, en sujetos concretos que valoran los objetos desde sus perspectivas y circunstancias singulares. Para todos estos teólogos, solamente si se asume la presencia de este rol de la subjetividad en la valoración económica es que puede entenderse adecuadamente la vida comercial, tanto en su dinámica propia como en su relación con la ética.

La literatura dedicada a estudiar estas cuestiones ha abordado esta problemática destacando que todos ellos apelaron a esta idea para oponerse a la objetivización del valor. Sería una especie de intuición común, compartida en general por la tradición salmantina. En esta dirección, por ejemplo, uno encuentra en la clásica obra de Schumpeter, que, hablando sobre estos autores, a quienes llama los "doctores escolásticos", explícitamente reconoce que "la distinción aristotélica entre valor de uso y valor de cambio se profundizó y desarrolló hasta desembocar en una teoría subjetiva o utilitarista del valor de cambio o precio"3. A lo largo de las páginas en las que analiza esta tradición de pensamiento, Schumpeter señala que hay diferencias y matices entre las ideas de cada uno de los “doctores escolásticos", pero que, sin embargo, la tesis central que reafirma la importancia del aspecto subjetivo presente en la valoración, es compartida en sus aspectos generales por todos ellos. Y así, su exposición se enfoca sobre todo en estos elementos comunes más que en las diferencias que pudiera haber entre ellos.

Contemporánea a Schumpeter, Grice Hutchinson realizó una lectura interpretativa de esta escuela muy similar. Ella señala que la "Escuela de Salamanca" tiene una teoría del valor marcadamente subjetivista ${ }^{4}$. Y este giro que admite cierta subjetividad presente en la valoración no se limita a servir como una mera descripción neutra de los actos de compra y venta, sino que también goza de cierta aprobación moral por parte de estos teólogos ${ }^{5}$. Como Schumpeter, Grice Hutchinson reconoce que no todos estos autores son igualmente subjetivistas en

2 Raymond de Roover, "Scholastic Economics: Survival and Lasting Influence from the Sixteenth Century to Adam Smith", The Quaterly Journal of Economics, LXIX, n. 2, (may-1955): 161190.

3 Joseph Alois Schumpeter, Historia del Análisis Económico (Barcelona: Ariel, 1971): 136. La cursiva es mía.

4 Marjorie Grice-Hutchinson, The School of Salamanca. Readings in Spanish Monetary Theory 1544-1605 (Oxford: Clarendon Press, 1952): 48

5 Marjorie Grice-Hutchinson, The School of Salamanca, 49: "From the tone of our writers it is evident that the subjective theory of value enjoyed their moral approval" 
su concepción del valor, encontrándose discrepancias entre unos y otros sobre cuál es el límite de esta subjetividad ${ }^{6}$. Incluso señala a Diego de Covarrubias, como uno de los más marcadamente subjetivistas ${ }^{7}$. A pesar del explícito reconocimiento de la existencia de estas discrepancias, en su trabajo la teoría del valor es abordada con una mirada global, sin entrar tampoco en los debates internos suscitados dentro de esta escuela. Unos pocos años después, Oreste Popescu también analizó y repasó las ideas de los maestros salmantinos y sus discípulos, asumiéndola como una posición más o menos homogénea y enfrentada a la objetivización del precio que suponía la teoría del valor basada en el costo de producción o "del valor trabajo", usualmente atribuida (equivocadamente) a Duns $\mathrm{Scoto}^{8}$.

Más recientemente, Odd Langholm señaló algo similar. Para él, hay que reconocer que la escolástica y luego la segunda escolástica sostuvieron que la estimación común ocupaba un lugar central si se quería conocer la naturaleza de los precios. Sin embargo, esta subjetividad presente en la economía debía ser matizada y contextualizada si se quería entender bien tanto el pensamiento económico como el fundamento filosófico que desarrollaron estos autores ${ }^{9}$. Un énfasis excesivo de cualquiera de los extremos, sea de la objetivización del valor, sea en su subjetivización, es, a su juicio, algo que desnaturaliza la concepción escolástica del valor económico ${ }^{10}$. El esfuerzo de Langholm se centra, entonces, en mostrar de qué manera, en líneas generales, todos estos teólogos entendieron que la valoración tenía elementos subjetivos y objetivos de un modo combinado. Evita, sin embargo, ingresar en los debates suscitados entre cada uno de ellos. También Alejandro Chafuén ha seguido una perspectiva parecida. En su trabajo desarrolló una presentación general del tema del valor económico. Tomando textos de los más reconocidos representantes de esta tradición, intentó demostrar que, más allá de las diferencias, las ideas de todos estos autores "deben considerarse como impulsoras y defensoras de la teoría subjetiva del valor"11. El mismo tipo de abordaje, resaltando el rol otorgado a la subjetividad dentro de la

\footnotetext{
6 Marjorie Grice-Hutchinson, The School of Salamanca, 48.

7 Marjorie Grice-Hutchinson, The School of Salamanca, 48.

8 Oreste Popescu, Aspectos analíticos en la doctrina del justo Precio en Juan de Matienzo (1520-1579) (Buenos Aires: Programa BIBLEH-UCA-CONICET, 1982), 96-97

9 Odd Langholm, The Legacy of Scholasticism in Economic Though (Cambridge: Cambridge University Press, 2006), 85.

10 Odd Langholm, The Legacy of Scholasticism in Economic Though, 85 y también especialmente 99.

11 Alejandro Chafuen, Raíces cristianas de la economía de Mercado (Santiago: Fundación para el Progreso, 2013), 201. Y es justo señalar que el propio Chafuén destaca a continuación que efectivamente hay diferencias entre la subjetivización presente entre los escolásticos y la que se encuentra entre los economistas del siglo XIX. La de los primeros serían solamente ideas "precursoras", pero no son "idénticas".
} 
Escuela de Salamanca asumida como una cierta tradición de pensamiento más o menos uniforme, puede verse en el reciente trabajo de Alves y Moreira ${ }^{12}$.

En unos de sus trabajos más conocidos, Gómez Camacho también abordó la temática con un enfoque centrado en esta tradición de un modo abarcativo. El eje de su interpretación pasa, como en los anteriores autores, en la centralidad otorgada a la noción de estimación común y al papel de la subjetividad. Es de destacar, sin embargo, que sobre el final del capítulo en el que trató más específicamente la temática del fundamento del valor económico, Gómez Camacho señaló que una revisión minuciosa de estos autores permite, en rigor, reconocer diferentes posiciones respecto del grado de importancia concedido a la subjetividad en la valoración económica ${ }^{13}$. Pero el tratamiento que hace de estas diferencias no pasa de ser una propuesta de trabajo a futuro.

Recientemente, Gómez Rivas ha destacado también este aporte de la "Escuela de Salamanca" a la teoría subjetiva del valor, intentando establecer lazos entre sus ideas económicas y desarrollos realizados en los siglos siguientes y fuera del ámbito hispánico. Por la perspectiva elegida por él al comienzo de su trabajo, las discusiones internas no han sido abordadas, en la medida en que el desafío propuesto por el autor fue el de intentar un diálogo entre diferentes tradiciones de pensamiento ${ }^{14}$.

Todo este intento por mostrar la presencia de este elemento subjetivo que la tradición escolástica destacó al reflexionar sobre la valoración económica, ha contribuido a mostrar su innegable modernidad y riqueza. Sin embargo, creo que, como una consecuencia no querida, este tipo de aproximación ha llevado a que sea concebida como una tradición con una homogeneidad y unidad interna, a mi juicio, muy exagerada. Probablemente, esta idea de homogeneidad se ha visto reforzada debido a un estilo de escritura muy similar, a lo que se suma una reiterada apelación a fórmulas y ejemplos casi idénticos. Todo ello ha contribuido, en mi opinión, a la invisibilización de un debate muy profundo y muy complejo en términos conceptuales, y que se dio entre algunos de los autores más reconocidos de este período. En mi opinión, una revisión minuciosa de los textos muestra que, de un modo sutil (a veces quizás extremadamente sutil, vista la autoridad a la que se cuestionaba) pero firme, cada uno de estos autores critica

12 Andre Azevedo Alves José Manuel Moreira, The Salamanca School (New York/London: Continuum, 2010) 71-73.

13 Francisco Gómez Camacho, Economía y Filosofia Moral: la formación del pensamiento económico europeo en la Escolástica Española (Madrid: Síntesis, 1998), 147.

14 León Gómez Rivas, "La teoría del valor en la Escuela de Salamanca", en Antonio Miguel Bernal Rodríguez, Modernidad de España (Madrid: Marcial Pons, 2017), 293-315. 
aspectos esenciales de la posición de los anteriores, y postula un cambio sustancial respecto a la naturaleza del valor económico.

En este artículo, en particular, me centraré en las posiciones de Tomás de Aquino, Conrado Summenhart, Domingo de Soto y Diego de Covarrubias y Leyva. A pesar de que, a primera vista, parecen sostener sustancialmente la misma posición respecto al fundamento filosófico del valor económico, destacándose el hecho de que cada uno de ellos va citando a los anteriores para defender sus propias ideas, una lectura atenta de los argumentos, y un desmenuzamiento exhaustivo de los ejemplos y casos concretos a los que apelan para demostrar sus ideas, muestran que esto no es así. Aun cuando sistemáticamente se comparen caballos, gemas, ratones y pulgas, la conclusión a la que llegan en cada caso es muy diferente. De este modo, en las páginas que siguen me propongo demostrar que, aun contra las apariencias que surgen de una lectura algo apresurada, entre el siglo XIII y el siglo XVI se desarrolló un complejo debate conceptual en un tema central de la filosofía de la economía, como es el del fundamento del valor económico. Al mismo tiempo, señalar que este debate oculto, antecede con varios siglos a complejas discusiones que se han dado sobre esta temática a partir sobre todo de la segunda mitad del siglo XIX y hasta nuestros días.

\section{LOS ANTECEDENTES: SAN AGUSTÍN Y ARISTÓTELES}

La tradición escolástica, en su aproximación a la cuestión de la valoración económica, apeló a dos textos que pueden ser considerados centrales, escritos por San Agustín y por Aristóteles ${ }^{15}$. Antes de avanzar con el debate escolástico, conviene revisar al menos las ideas centrales que se desprenden de ellos.

El referido texto de San Agustín fue tomado de la Ciudad de Dios y fue luego constantemente citado a lo largo de todo el período medieval y temprano moderno. Decía allí el Hiponense, hablando sobre el precio: "existen otras muchas suertes de apreciación según la utilidad de cada uno; así sucede que anteponemos algunos seres que carecen de sentido a otros que lo tienen (...) ¿Quién no prefiere tener en su casa pan a tener ratones; dinero a tener pulgas?"16.

Como se puede ver, para San Agustín una mirada honesta sobre el mundo del comercio muestra que el valor económico de los objetos no puede ser

15 Los primeros capítulos de la obra de Langholm abordan esta cuestión. Odd Langholm, The Legacy of Scholasticism in Economic Though, capítulos 1, 2 y 3.

16 San Agustín, De Civitate Dei, XI, XVI (Tomo la traducción de San Agustín, La Ciudad de Dios ( $\left.1^{\circ}\right)$ (BAC, Madrid, 2007): 713). 
deducido de su naturaleza o del grado que ocupa en la escala de los seres. Aun cuando uno intentara hacer un esfuerzo argumentativo, la fuerza de la experiencia es tal que no queda otra opción que reconocer que, al menos en el ámbito de la vida comercial, la mayor o menor valoración no puede provenir de allí. En orden a encontrar una justificación alternativa, el Hiponense señala que el valor económico está vinculado a la "utilidad de cada uno". Sin este componente subjetivo no se entendería la realidad del comercio que cotidianamente se puede ver en las aldeas y comunidades, donde ratones y pulgas valen menos que el dinero y el pan.

Aristóteles proveyó a la escolástica de un segundo texto especialmente relevante para esta temática. En efecto, el Estagirita analizó la cuestión del fundamento del valor económico en el Libro V de la Ética Nicomaquea. Allí señaló que: "Todas las cosas, por lo tanto, deben ser medidas por una, como se ha dicho antes. En realidad de verdad esta medida es la chreía, la cual mantiene unida a todas las cosas", y más adelante agrega "Mas por una convención la moneda ha venido a ser el medio de cambio representativo de la chreía" 17 .

Este texto contribuyó a la polémica sobre el fundamento del valor no solamente por la tesis que presentaba, según la cual la chreía es aquello que en última instancia mide el valor económico, sino también por la misma dificultad que encerraba la traducción del término del griego al latín. En efecto, la noción de chreía puede ser traducido como "indigencia", que hace hincapié sobre todo en la "necesidad" que tiene el sujeto del bien, pero también en "uso" o "utilidad", que hace referencia a las propiedades que tiene el bien y que satisfarán a quién lo adquiera. La complejidad de la palabra original griega, que de alguna manera contiene los dos matices, hará difícil a los traductores medievales del Estagirita encontrar un término preciso a la hora de volcarlo al latín ${ }^{18}$. Por otra parte, Aristóteles hace un claro reconocimiento de que, en la vida social económica, el valor se manifiesta, en la práctica, en el precio monetario que tienen los bienes. El precio no es el valor del objeto, sino que es el camino por el cual podemos representarnos su valor y posibilitar de una manera más eficaz el intercambio.

17 Aristóteles, Ética Nicomaquea, Libro V, 1133 A. Tomo la traducción de Antonio Gómez Robledo como base pero dejando transliterado el texto griego chreía. Aristóteles, Ética nicomaquea, versión española y notas de A. Gómez Robledo. (México: Universidad Nacional Autónoma de México, 1983). He revisado también Aristotle, The Nichomachean Ethics with an english translation by $H$. Rackham (Cambrigde, MA: Harvard University Press, 1994).

18 Este problema ha sido señalado por numerosos autores. Por ejemplo, Alejandro Chafuen, Raíces cristianas de la economía de Mercado, 186. Antes que él, ya lo había señalado también Oreste Popescu, Aspectos análiticos en la doctrina del justo Precio en Juan de Matienzo, 26-29. Recientemente Joel Kaye, Economy and Nature in the Fourteenth Century (Cambridge: Cambridge University Press, 2004), 68 y ss. 
Y este intercambio, además de ser conveniente para las partes, debe ser también "justo". De allí la importancia que tiene para la ética aristotélica comprender cuál es el fundamento último del valor. Justicia e intercambio económico están (o deberían estar) íntimamente unidos.

Estos textos de San Agustín y Aristóteles sirvieron durante todo el período escolástico (y luego de la Segunda Escolástica) como marco conceptual de la discusión. Es siempre apelando a su autoridad que se reafirmará una y otra vez que el precio de los objetos no puede estar atado a los grados de ser, y que, en cambio, había que mirar al uso que se hacía de ellos si se quería realmente entender su fundamento último. Uno encuentra esta fórmula repetida una y otra vez a lo largo de diversas sumas y opúsculos, y los ejemplos a los que se apela para explicarla son notablemente similares. Sin embargo, a pesar de que todos asumieron esta necesidad de dar una explicación que conjugue al aspecto subjetivo que trae consigo la noción de "estimación", y el aspecto objetivo que exige la necesidad de que el precio sea "justo"19, el resultado obtenido en los diferentes casos fueron posiciones intelectuales muy diferentes entre sí.

\section{EL INICIO DE LA POLÉMICA: TENSIONES EN DOS TEXTOS DE TO- MÁS DE AQUINO}

Tomás Aquino aborda la cuestión del fundamento último del valor económico especialmente en dos textos, que, por la influencia que tuvieron sobre autores posteriores (y especialmente, sobre los que trataremos a continuación), merecen una especial atención. El primero de ellos se puede ver en su comentario al libro V de la Ética de Aristóteles. Y el segundo es el que presenta en la Suma de Teología, específicamente en la q. 77 de la II-IIae, particularmente en su artículo 1. Una lectura de ellos, y especialmente, una comparación entre uno y otro, ya anticipan parte de lo que sucederá en los siglos siguientes.

El primero en el que quisiera centrarme es el que se puede leer en el texto en que comenta la Ética a Nicómaco. Como se dijo, en la obra del Estagirita el análisis se centraba no solamente en la naturaleza del precio, sino en la del "precio justo", en orden a que el comercio que surja de él lo sea. Puesto a comentarlo, Tomás sostiene sin dudar que, como señala Aristóteles, si no hubiera indigencia, no habría intercambio de bienes en la sociedad ${ }^{20}$. Y un poco más adelante,

19 Bernard W. Dempsey, "Just Price in a Functional Economy", The American Economic Review, 25 (1935): 485

20 Tomás de Aquino, In decem libros Ethicorum Aristotelis ad Nicomachum expositio (Torino: Marietti, 1964), pto. 981. 
aborda la temática del fundamento último del valor, que sería aquello que nos permite medir los intercambios, y que, una vez conocido, nos permite aspirar a lograr cierta equidad en ellos. El dominico se apoya con firmeza en el texto de Aristóteles y, sin dudar, dice que es la misma indigencia lo que verdaderamente mide todo intercambio. Es ella (la indigentia) la que mide el valor de las cosas al momento de comprar, vender o trocar, siendo el dinero algo que se establece para el mismo $\mathrm{fin}^{21}$. Finalmente, cerrando la cuestión, el Aquinate señala que el precio de las cosas depende de la indigencia, pero esta a su vez es una indigencia en relación al "uso" humano de esas cosas: "el precio de las cosas se impone según la necesidad (indigent) que tengan los hombres de ellas para su uso (ad suum usum)"22. En otras palabras, esta "indigencia", que nos hace valorar más o menos determinadas cosas, se manifiesta, si cabe la expresión, en la necesidad de uso que tenemos de los bienes. Para culminar su explicación, el Aquinate completa lo dicho señalando que el valor económico no puede ser un resultado del grado de ser de los objetos. Para demostrar ello recurre al ejemplo de San Agustín y señala que si valoráramos en función de la naturaleza de los objetos en cuestión "un ratón, que es un animal sensible, debería tener mayor precio que una perla, que es inanimado" 23 .

En la II-IIae, q. 77 a. 1 uno encuentra alguna diferencia que a primera vista parece menor, pero acaba siendo muy importante en términos conceptuales. En ese artículo, el centro de la discusión es si es lícito vender una cosa a más de lo que vale. Para resolver esto, uno de los puntos a dilucidar es precisamente el valor de las cosas, pues solamente luego de conocido el valor es que puede saberse si se está vendiendo por encima de él. En su análisis de la cuestión, su reflexión se vuelve muy concreta. Al igual que en cuando comenta la Ética a Nicómaco, el Aquinate señala que es la indigencia la que mueve a hacer los intercambios. Pero al momento de especificar la cuestión de los precios en sí, el texto cambia levemente. Dice allí el dominico que, en primer lugar, es el precio el elemento que muestra el valor del objeto y, a continuación, señala que este, en última instancia, está basado en el uso (usum) que dan los hombres a esos

21 Tomás de Aquino, In decem libros Ethicorum Aristotelis ad Nicomachum expositio, pto. 981: "hoc autem unum, quod omnia mensurat secundum rei veritatem est indigentia, quae continet omnia commutabilia, in quantum scilicet omnia referuntur ad humanam indigentiam". Más adelante señala algo similar (pto. 985) "Primo ostendit quod necessitas sit mensura secundum rei veritatem. Secundo quomodo denarius sit mensura secundum legis positionem".

22 Tomás de Aquino, In decem libros Ethicorum Aristotelis ad Nicomachum expositio, pto. 981: "rebus pretia imponuntur, secundum quod homines indigent eis ad suum usum".

23 Tomás de Aquino, In decem libros Ethicorum Aristotelis ad Nicomachum expositio, pto. 981: "alioquin unus mus, quod est animal sensibile, maioris pretii esset quam una margarita, quae est res inanimata" 
bienes $^{24}$. Como se puede ver, el Aquinate ha hecho un casi imperceptible movimiento de la noción de "indigencia" a la de "uso" y, de este último, al precio concreto en que efectivamente se realizan los intercambios. Ahora directamente es el "uso" de los bienes el que acaba siendo el elemento que permite comprender el valor de las cosas. Este uso, a su vez, deberá ser "prudente" y, en ese sentido, es este uso el que efectivamente mide algo para que sea un precio justo.

Para completar lo anterior, en la misma cuestión, pero ahora en el artículo segundo, se apela a lo dicho por San Agustín. Con esto, lo que busca es señalar que la valoración económica no puede quedar exclusivamente atada a la pura naturaleza del objeto, pues la misma experiencia nos muestra lo absurdo de esa posición: en la vida económica un caballo vale más que un siervo, cuando está claro que desde el punto de vista de la dignidad esto no es asi ${ }^{25}$. Pero, al mismo tiempo, en la misma respuesta también señala que sí es necesario conocer ciertas cualidades del objeto para que la venta sea efectivamente justa. Haciendo esto, el Aquinate vuelve a llevar la atención sobre el objeto, no ya en su naturaleza metafísica, pero sí en sus cualidades, especialmente aquellas que están en relación con el uso que pretende hacer quien compra. Con esta precisión, pareciera que el doctor angélico establece algún tipo de marco que objetive algo la valoración económica si no se quiere caer en una subjetivización total del precio.

De esta manera, Tomás presenta en sus textos dos visiones que no son totalmente equivalentes, aun cuando en ambos casos sea la estimación la que ocupe el punto de partida para comprender a la valoración económica. En el primer caso, la estimación remite a la "indigentia" que tiene el sujeto, es decir, a la necesidad que tiene de usar las cosas. La referencia al "uso" aparece luego de esta necesidad presente en los hombres. En cambio, en el segundo texto, si bien la necesidad es la que genera los intercambios, al precisar la naturaleza de los precios el texto del Aquinate muestra a la estimación remitiendo directamente al "uso" o a la "utilidad" que se hace del objeto. La indigencia no es ahora mencionada como un elemento central, como lo había sido en el Comentario a la Ética a Nicómaco. De un modo sutil, diera la impresión de que en la Suma de Teología la centralidad se ha trasladado del sujeto que valora y necesita algo al objeto a adquirir y la utilidad que este da (o podría dar). Pero en

24 Tomás de Aquino, Summa Theologiae II-IIae, q. 77, a. 1, sol: "Quantitas autem rerum quae in usum veniunt mensuratur pretium datur".

25 Tomás de Aquino, Summa Theologiae II-IIae, q. 77, a. 2, ad 3: “Ad tertium dicendum quod, sicut Augustinus dicit, in XI de Civ. Dei, pretium rerum venalium non consideratur secundum gradum naturae, cum quandoque pluris vendatur unus equus quam unus servus, sed consideratur secundum quod res in usum hominis veniunt". 
ambos casos, como se ve, es la estimación la que acaba siempre remitiendo al uso o utilidad del bien, siendo por lo tanto la valoración económica una combinación surgida del sujeto que estima y el objeto que habrá de ser usado por él.

\section{CONRADO SUMMENHART Y LA UTILIDAD COMO FUENTE DE VALORACIÓN}

En el siglo XV, Conrado Summenhart abordó la misma cuestión en su obra "De Contractibus". Allí, en la Q. LVI del Tractatus III, específicamente se pregunta por el conocimiento del valor de las cosas que se venden o se comercian.

Lo primero que aparece aclarado es que el valor, al menos en términos económicos, es revelado por el precio ${ }^{26}$. Y por lo tanto, lo central, si se quiere que haya un trato justo, es que el precio sea igual al valor del objeto, y no mayor que él ${ }^{27}$. Ahora bien, conocer el valor de algo, señala Conrado, implica primero reconocer que todas las cosas tienen un doble valor. El primero de ellos es el valor natural. Este es el resultado del grado de ser de cada objeto, y será mayor el valor de algo cuanto mayor sea su perfección ${ }^{28}$. El segundo es el que se basa en el uso que hacen las personas de los objetos ${ }^{29}$. En concordancia con la tradición anterior, Conrado señala que en la vida económica el valor que hay que tener en cuenta es el valor del uso del objeto y no su valor natural.

En orden a probar esto, Conrado apela al ejemplo agustiniense. Desde el punto de vista del valor natural, la mosca, la hormiga y la pulga valen más que una gema ${ }^{30}$, pero desde el punto de vista del uso tienen mayor valor el vino y el pan que la mosca, la hormiga y la pulga. Y los hechos muestran, además, que en la compra y venta se utiliza esta segunda forma de valorar; caso contrario, se

26 Conradus Summenhart, De contractibus licitis atque illicitis (Venecia: Bernardum Iuntam, 1580), Tract. III, q. LVI, 259: "quia cognoscere quantitatem valori mercis conducit ad cognoscendum an precium sit sibi equale vel maius". También puede verse en la misma página, pero en la columna 1.

27 Conradus Summenhart, De contractibus licitis atque illicitis, Tract. III, q. LVI, 259, "Nam si precium est aequale valori mercis, potest illud licite exigere, si est maius, non potest".

28 Conradus Summenhart, De contractibus licitis atque illicitis, Tract. III, q. LVI, "Ille est perfectio essentialis rei consistens in gradu specifico, per quem res constituitur in esse specifico, quo valore illa res est validior, quæ est perfectionis essentiae secundum speciem: puta cuius differentia specifica est perfectior, vel etiamsi est totum compositum, cuius forma substantialis specifica est nobilior".

29 Conradus Summenhart, De contractibus licitis atque illicitis, Tract. III, q. LVI, 259. “Alius est valoris usualis"

30 Conradus Summenhart, De contractibus licitis atque illicitis, Tract. III, q. LVI, 259. 
podría "exigir más por un ratón que por una gema preciosa"31. Cómo se puede ver, Conrado parece presentar una especie de repetición de lo dicho por Tomás: en el mundo económico estimamos los bienes en virtud del uso que hacemos de ellos y no en virtud de la naturaleza, y es tanto la autoridad de Agustín como la experiencia la que nos lo muestra. Pero Conrado agrega, además, una argumentación que, al menos a mi juicio, muestra una ironía exquisita. Para completar su demostración señala que si el valor dependiera del grado de ser, los comerciantes deberían ser grandes conocedores de la física y la metafísica, para así poder conocer mejor la naturaleza de sus bienes y exigir el precio justo ${ }^{32}$.

El problema aparece cuando uno intenta comprender qué entiende Summenhart por "uso" de un objeto. En su reflexión, uno encuentra que el "uso", si bien no alude a la naturaleza "metafísica" ni "física" de un objeto, sin embargo, sí se refiere a características objetivas de los bienes. Esta utilidad que hace que valoremos los bienes no es una cuestión de percepciones relacionadas con la subjetividad de las personas, sino que, por el contrario, es una propiedad o una suma de propiedades poseídas por el objeto a comprar o vender ${ }^{33}$. Y un poco más adelante señala, en el mismo sentido, que el valor estaría asociado a la capacidad que tenga el objeto de satisfacer "la utilidad y comodidad (utilitatem et commoditatem) que los hombres desean conseguir con esos bienes", y que, por lo tanto, para conocer el precio hay que conocer bien la capacidad que tiene ese bien de proporcionar esa comodidad (commoditatem) ${ }^{34}$. Finalmente, esto es reiterado más adelante cuando señala que esa comodidad que objetivamente puede brindar el bien es algo que puede ser mejorado por parte del vendedor, y que, al hacerlo, hará que aumente necesariamente su valor económico ${ }^{35}$. Uno es el fundamento último del otro. La formulación podría ser resumida de la siguiente manera: a mayor utilidad objetiva del bien habrá siempre mayor valor económico.

Como se puede ver, Summenrhart acepta que la estimación que nos hace conocer el valor es aquella que refiere la necesidad de uso o de utilidad que hay del algún bien. Sin embargo, esta utilidad es entendida como una propiedad

31 Conradus Summenhart, De contractibus licitis atque illicitis, Tract. III, q. LVI, 260: "consequens est falsum: tunc pro mure posset plus exigere, quam pro gemma preciosa"

32 Conradus Summenhart, De contractibus licitis atque illicitis, Tract. III, q. LVI, 260: "si requireretur, oporteret, venditores esse magnos physicos et metaphysicos, ut cognoscerent quam perfecta esse merx in gradu specifico".

33 Conradus Summenhart, De contractibus licitis atque illicitis, Tract. III, q. LVI, 259: "tanto res est validior, quanto ad usum humanum habilior".

34 Conradus Summenhart, De contractibus licitis atque illicitis, Tract. III, q. LVI, 260

35 Conradus Summenhart, De contractibus licitis atque illicitis, Tract. III, q. LVI, 260: "quia quanto magis meliorata est merx, tanto validior est." 
natural del bien, es decir, como algo que puede ser mejorado o disminuido por el artífice o incluso por el vendedor, o también, que siendo poseída por el objeto desde siempre, es por diferentes motivos conocida (o descubierta) por las personas luego de transcurrido cierto tiempo ${ }^{36}$. En uno u otro caso ya hay algo presente objetivamente en el bien que es previo a la estimación. De este modo, se observa que Conrado, luego de lo que puede considerarse un primer momento "subjetivista", que se da cuando el valor se lo asocia con el "uso", vuelve a “objetivizar" el valor económico al quedar ahora atado ya no a su naturaleza metafísica, pero sí a propiedades y características del mismo bien con el que se desea comerciar y que son independientes de la percepción de los sujetos.

\section{DOMINGO DE SOTO Y LA INDIGENCIA}

El dominico Domingo de Soto aborda la cuestión en su obra De Iustitia et Iure, de un modo especial en el libro $\mathrm{VI}^{37}$. Una primera aproximación al texto muestra una estructura que parece idéntica a la de Tomás y a la de Conrado. Preguntándose cuál es el fundamento de la estimación de los precios, Soto señala que estos no pueden basarse en la naturaleza de las cosas, sino que hay que mirar la utilidad que los objetos dan a los hombres: "El precio de las cosas no debe estimarse (aestimanda sunt) según la naturaleza de ellas, sino por la utilidad que prestan a los hombres (in usus veniunt humanos)"38. A continuación, también Soto apela a la autoridad de San Agustín y a la experiencia cotidiana que se vive en el mercado. Citando al Hiponense, señala que "más quería el hombre tener trigo en casa que ratones"39. Y luego destaca que "es costumbre vender una casa en un precio mayor que un caballo; y a veces un caballo que un criado, a pesar de que no haya nada de lo corporal que pueda igualarse con la naturaleza del hombre" ${ }^{40}$. En resumen, el valor está asociado al uso y este no responde a la naturaleza del objeto.

36 Conradus Summenhart, De contractibus licitis atque illicitis, Tract. III, q. LVI, 262-3.

37 Las citas serán tomadas de Domingo de Soto, De Iustitia et Iure. Libri Decem/De la justicia y el derecho en diez libros, edición facsimilar de la edición de 1556, traducida por Marcelino González Ordóñez O.P., Vol. III, (Madrid: Instituto de Estudios Políticos, 1968). Los textos en castellano que sean citados en el cuerpo del trabajo, de no mediar aclaración, serán tomados de la traducción propuesta en esta edición.

38 Domingo de Soto, De Iustitia et Iure, L. VI, q. II, a. 3, 546: "Pretia rerum non secundum ipsarum naturam aestimanda sunt, sed quatenus in usus veniunt humanos"

39 Domingo de Soto, De Iustitia et Iure, L. VI, q. II, a. 3, 547.

40 Domingo de Soto, De Iustitia et Iure, L. VI, q. II, a. 3, 547: "Et solet domus plus vaenire, quam equus, et nonumpuam equus, quam seruus: licet cum natura hominis nihil in corporalibus conferri ad aequalitatem valeat". 
El conflicto aparece cuando uno se lanza comprender qué es lo que para Soto vuelve útil a los objetos, de manera que luego las personas lo valoren más o menos según el caso. Aquí el dominico decide apelar a la Ética de Aristóteles y señala que esta estimación de la utilidad tiene como fundamento último a la necesidad (indigentia) que los hombres tienen de los bienes. En otras palabras, el valor depende de la utilidad, pero en Soto esta tiene su razón última en la necesidad que los hombres tienen de las $\operatorname{cosas}^{41}$. Por eso, señala Soto, "Por lo tanto, debemos admitir que los precios deben medirse según la necesidad (indigentia)" $^{\prime 42}$. Y, por lo tanto, para comprender el fundamento del valor, hay que mirar en primer lugar a la necesidad ${ }^{43}$.

Este traslado conceptual que lo hace a Soto ir de la noción de uso y de utilidad a la noción de indigencia o necesidad del sujeto lo lleva a cuestionar muchas de las ideas que habían sido sostenidas por Conrado. Y, en particular, uno encuentra que Soto específicamente señala que el valor no puede ser el resultado de la utilidad del objeto considerado por sí mismo, como había señalado el primero. Para oponerse a planteamientos objetivistas como el de Summenhart, Soto propone distintos argumentos. La tesis central que siguen estos argumentos, sin embargo, radica en la afirmación de que la utilidad de un objeto, la cual hace que el comprador desee adquirirlo, no es algo propio del bien, sino que está en consonancia con el uso que se quiere hacer de él. "Por ejemplo", dice Soto, "cuando uno compra un caballo necesario para la guerra al que lo compra, pero que no lo es para el que lo vende" ${ }^{44}$. En otras palabras, quien compra atiende al uso que él le dará una vez que lo adquiera, y no al uso del que hacía el vendedor (o al menos, no necesariamente coinciden uno y otro). Así, al estar referido el uso a la necesidad del sujeto que compra y no en el bien, no hay forma de que el vendedor lo incluya lícitamente en el precio, pues él es dueño del objeto "mas la utilidad que recibe el comprador no es del vendedor" más, si el precio se fijara atendiendo a la utilidad objetiva que los bienes dan a quienes producen remedios necesarios para la salud estos podrían cobrar virtualmente lo que quisieran ${ }^{46}$.

41 Domingo de Soto, De Iustitia et Iure, L. VI, q. II, a. 3, 546.

42 Domingo de Soto, De Iustitia et Iure, L. VI, q. II, a. 3, 546: "Ergo indigentia admetiri debemus rerum pretia"

43 Domingo de Soto, De Iustitia et Iure, L. VI, q. II, a. 3, 547.

44 Domingo de Soto, De Iustitia et Iure, L. VI, q. 3, a. 1, 550.

45 Domingo de Soto, De Iustitia et Iure, L. VI, q. 3, a. 1, 550. "Commodum autem non erat res vendentis". La utilización del término "commodum" tiene una evidente resonancia a lo dicho por Conrado, a quien claramente está cuestionando.

46 Domingo de Soto, De Iustitia et Iure, L. VI, q. 3, a. 1, 550. Es de señalar que un argumento muy similar ya había sido sostenido antes por Pedro Juan Olivi, De Contractibus, q. 2, pto. 24. En la 
En cambio, dice Soto, si uno mira a la necesidad como elemento central, la cuestión queda para él mejor explicada. Cuanto más necesite el vendedor vender, menor será el precio, y por el contrario, cuanto más sean los compradores y más necesitan del bien, mayor será el precio ${ }^{47}$.

De esta manera, si Conrado consideraba que las personas estimaban en virtud de la utilidad objetiva del bien, Soto, por el contrario, tiene una mirada claramente más subjetivista, en la medida en que ahora el centro de la explicación lo ocupa la indigencia que las personas tienen de ellos. Este giro hacia la indigencia como fundamento, sin embargo, no termina de ser un giro hacia la subjetividad total del valor económico. Aun cuando el eje ha pasado del objeto al sujeto, la referencia no es la necesidad del sujeto particular, sino la necesidad del hombre considerado como miembro de una comunidad particular, y que estima en el marco de una realidad concreta ${ }^{48}$. Este último movimiento argumentativo supone ahora cierta objetivización del valor. Soto reiteradamente destaca la idea de que con su apelación a la indigentia no está refiriéndose a necesidades privadas de un individuo particular sino aquellas que pueden ser reconocidas como necesidades comunes ${ }^{49}$. Esta necesidad, en tanto que es común a todos los miembros de la comunidad, explica por qué, dada la situación de abundancia o escasez que allí se viva, el precio aumentará o disminuirá respectivamente ${ }^{50}$. Y de hecho, esta objetivización de la necesidad le permite justificar a Soto la potestad de la ciudad y de los gobernantes de establecer los precios, si no los de todos los bienes, al menos de los más relevantes en términos comunitarios $^{51}$. Al no ser una necesidad exclusiva e individual sino la del hombre en cuanto miembro de una comunidad concreta la que sirve de fundamento del valor, entonces una persona prudente (idealmente, el gobernante) podría

edición Pierre de Jean Olivi, Tratado de los Contratos (Estudio preliminar de R. Ramís Barceló y traducción de P. Ramís Serra y R. Ramís Barcelo) (Madrid: Dykinson, 2017), 85.

47 Domingo de Soto, De Iustitia et Iure, L. VI, q. 2, a. 3, 548.

48 Domingo de Soto, De Iustitia et Iure, L. VI, q. 2, a. 3, 548.

49 Domingo de Soto, De Iustitia et Iure, L. VI, q. 2, a. 3, 548: "Ubi habetur precia rerum non ex affectu aut utilitate singulorum sed communiter fungi. Hoc est, non secundum privatorum existimationem, sed secundum communem prudentiam constitui”. También puede verse L. VI, q. 2, a. 3,549 .

50 En mi opinión, la abundancia y escasez en relación con el precio, tema que ha atraído a los estudiosos de Soto, debería verse sobre todo en línea con lo anteriormente dicho sobre la centralidad de la indigencia. La abundancia y la escasez en el fondo son entendidas por él, a mi juicio, como necesidades compartidas, y eso hace que aumente o disminuya el valor de las cosas según si hay una o la otra. Si esta interpretación es correcta, al tratar sobre la abundancia y escasez parece tender más a objetivar el precio que a subjetivarlo. Un desarrollo de esta idea excede el presente trabajo.

51 Domingo de Soto, De Iustitia et Iure, L. VI, q.2, a. 3, 547: "Notandum est quod meritailla et causas statuendi rerum pretia existimare, per se quidem primum ad rem publicam et eius gobernatores spectac". También puede verse L. VI, q.2, a. 3, 549. 
conocer mejor la necesidad real que hay en esa ciudad de ciertos objetos y darles así un precio justo, que luego debería ser aceptado por el resto de la comunidad.

Como señalara Garrán Martínez, es cierto entonces que Soto apela a la utilidad que reportan los bienes como fundamento del valor económico, pero una lectura completa de los textos obliga a hacer, al menos, "algunas puntualizaciones" 52 , que lo alejan de la excesiva subjetividad con que cuenta hoy en día, particularmente en ámbitos de la filosofía de la economía, la noción de "utilidad". La excesiva subjetivización del valor, librando la estimación a los intereses de cada individuo conlleva para él riesgos para la comunidad en su conjunto que es mejor evitar ${ }^{53}$.

\section{DIEGO DE COVARRUBIAS Y LA ESTIMACIÓN COMO FUNDAMENTO DEL VALOR ECONÓMICO}

La posición de Covarrubias dará una nueva perspectiva al debate. Siguiendo lo dicho por sus maestros y antecesores, Covarrubias señala también que el valor económico surge de la estimación y no de la naturaleza de los objetos. A esto se le suma el especial énfasis que él pone en señalar que cuando se habla de la estimación no se hace referencia a la estimación que hace una persona singular sino a la estimación de una comunidad de hombres ${ }^{54}$. Este énfasis por la "estimación común" parece, a primera vista, acercarlo a las ideas de Domingo de Soto.

El planteamiento de Covarrubias se vuelve sin embargo más complejo en la medida en que da un paso más allá y se pregunta qué hace que las personas estimen lo que estiman. Para resolver esto aparecen dos elementos que llaman notablemente la atención, y que muestran un mayor peso del aspecto subjetivo

52 José María Garrán Martínez, "La justicia en el ámbito mercantil según Domingo de Soto", Ciencia Tomista CXXXII (2005): 124. En este sentido, concuerdo también con la apreciación hecha por A. del Vigo Gutiérrez acerca de una cierta desconfianza por parte de Soto a admitir una valoración privada y subjetiva. El problema es que del Vigo Gutiérrez en su obra no lo dice refiriéndose particularmente a Domingo de Soto, sino que lo extiende de un modo general a "los escolásticos", generalización con la que no concuerdo, como ya he señalado. A. del Vigo Gutiérrez, "La teoría del justo precio corriente en los moralistas españoles del Siglo de Oro", Burgense: Collectanae Scientifica 20, 1 (1979): 57-130. Mírese por ejemplo 99.

53 Sin embargo, parece difícil sostener lo que dice Gordon sobre Soto. Según él, esto lo termina llevando de una posición originalmente subjetivista a una que basa el valor en los costos de producción. No concuerdo en absoluto con esa lectura. Barry Gordon, Economics analysis before Adam Smith (Londres: Macmmillan Press, 1975), 237.238. La posición de Garrán Martínez parece más ajustada al sentido de los textos.

54 Diego de Covarrubias y Leyva, Variarum ex iure pontificio, regio, et caesareo resolutionum libri III, (Lugduni: Sebastianum Bartolomaei Honorati, 1557), 398: "Iustum cuiusque rei precium non cuiuslibet affectione, aut sumptu constat: sed ex communi hominum estimatione perpenditur". 
como elemento central en la valoración. La primera es la afirmación que hace Covarrubias en la que señala que el precio justo no surge de la naturaleza de las cosas sino de la estimación, para destacar luego que esto es así aún cuando esta estimación sea "vana" 55 . En línea con la tradición, Covarrubias separa el precio de la naturaleza de las cosas y la asocia con la estimación, pero llamativamente acepta que una estimación vana pueda ser asociada a un precio justo. Es cierto que no sería la estimación insana de un individuo particular sino la de una comunidad de hombres. Sin embargo, esta tesis abre la posibilidad de que estimaciones poco racionales o poco prudentes den como resultados valores aprobados (o aprobables) en términos de justicia.

La segunda cuestión que llama la atención se ve al momento de revisar los ejemplos que usa Covarrubias para explicar mejor su posición. Es aquí donde se ve con más fuerza el importante peso que da él a la subjetividad, criticando a través de ellos las posiciones de los pensadores que lo antecedieron, en los que percibe lo que considera una equivocada objetivización del valor. Retomando una vez más el ejemplo de Agustín para reforzar la tesis de la separación entre valoración objetiva y valoración económica, dice Covarrubias que estos son claramente diferentes, pues si fueran idénticos habría que estimar "un caballo más que una gema" 56 . El texto es particularmente interesante por los matices que presenta, por lo que merece ser citado en toda su extensión: "Si hubiese que observar a la naturaleza de la cosa, habría que estimar a un caballo más que a una gema, por la utilidad del caballo, y porque todo ser vivo y animado es, por su naturaleza, más preciado que uno muerto e inanimado" $"$.

Como se puede ver, Covarrubias dice que la naturaleza del caballo es superior a la gema tanto por ser un viviente como por ser más útil que ella. Y, sin embargo, económicamente hablando, vale menos que la gema. En otras palabras, desde su perspectiva, una genuina separación entre el valor económico y el valor natural del objeto implica no solamente pensar al precio como algo independiente del grado de ser del bien sino también, lo que es más importante, pensarlo independiente de la utilidad propia y objetiva que damos a ese bien.

55 Diego de Covarrubias y Leyva, Variarum ex iure pontificio, regio, et caesareo resolutionum libri III, 399: "Primum, in contractibus emptionum, et venditionum, similibusque permutationibus, nequaquam attendi nec constitui iustum pretium ex natura rei, sed ex hominum estimatione, tamensi insana sit estimatio". El énfasis es mío.

56 Diego de Covarrubias y Leyva, Variarum ex iure pontificio, regio, et caesareo resolutionum libri III, 399

57 Diego de Covarrubias y Leyva, Variarum ex iure pontificio, regio, et caesareo resolutionum libri III, 399. El énfasis el mío. ("Nam si natura rei foret observanda, pluris esset estimandus equus quam gemma, ob utilitatem equi, et quod omne viuum et animatum pretiosius est mortuo et inanimato ex natura sua"). 
Siguiendo con el ejemplo, para Covarrubias concebir al precio como algo que se sigue de su naturaleza no implica solamente el pensar al precio como asociado al grado de ser, sino también el pensar el precio en función de lo útil que el bien puede resultar ${ }^{58}$. En el ejemplo, esto implica que es un error pensar que el caballo vale más que la gema por ser un viviente, como también pensar que éste, por servir, por ejemplo, para trabajar o para ir a la guerra, es decir, por ser más útil, debe valer más. Incluso si fuese útil para la comunidad, tampoco serviría como fundamento último del valor. Es un hecho indiscutible que usualmente el caballo vale menos que la gema, aun cuando se pueden hacer con él más cosas que con ella. De esta manera, cuando se remite el fundamento de la estimación a la utilidad o al uso que hacemos de las cosas, para Covarrubias también se está concibiendo al valor como algo ligado a la naturaleza del objeto, aun cuando no sea ahora la naturaleza entendida como grado de ser. Esta forma de sutil objetivización del valor económico también debe ser cuestionada y evitada. Quienes piensan a la estimación como algo ligado al uso, o a necesidades objetivas, están todavía confundiendo al valor económico con el valor surgido de la naturaleza del objeto, cuando en realidad son diferentes y, sobre todo, independientes uno del otro.

En este sentido, Covarrubias hace una clara revalorización del rol de la estimación, pero ahora considerada por sí misma. Es la estimación de los hombres de una comunidad la que termina dando un precio, pero parece imposible ir más allá en la búsqueda de un fundamento último de ella. En otras palabras, ya no es la utilidad objetiva del bien el fundamento de la valoración, ni la necesidad presente en los hombres: es la propia estimación humana acerca de ello lo que explica los precios. Incluso, como se dijo, esta estimación puede ser vana o irracional. Pareciera que tiene razón Grice Hutchinson, cuando al abordar la cuestión del fundamento del valor económico, señalaba a Covarrubias como uno de los más extremos subjetivistas dentro de esta escuela ${ }^{59}$. Sin embargo, aunque es cierto que el fundamento deja de estar en el objeto y pasa al sujeto que estima, tampoco hay en él una subjetivización total del valor económico, en la medida en que esta estimación hace siempre referencia a una estimación común y no una estimación aislada e individual ${ }^{60}$.

58 No deja de ser un dato interesante el que cite precisamente a Conrado, para luego decir casi lo contrario a lo dicho por él.

59 Marjorie Grice-Hutchinson, The School of Salamanca, 48

60 Diego de Covarrubias y Leyva, Variarum ex iure pontificio, regio, et caesareo resolutionum libri III, 399: "In precii iusti aestimatione non ese considerandum quanti res ipsa empta fuerit: nec quot labores pro eius adquisitione venditor fuerit perpessus: sed tantum habendam esse rationem communis hominum aestimationis". 


\section{CONCLUSIONES. UN DEBATE OLVIDADO}

Como decía al comienzo, entre los estudiosos de las ideas económicas de la escolástica y la segunda escolástica española hay un reconocimiento de la presencia de un elemento subjetivo en la teoría de la valoración. En orden a destacar este elemento subjetivo frente a aquellos que erróneamente atribuían a la escolástica una teoría del valor económico fundada en el trabajo, los diferentes matices y perspectivas propuestos por cada uno de estos teólogos parece haber quedado opacado y silenciado. Seguramente, también el uso de ejemplos comunes en todos ellos (ratones, caballos, gemas y moscas son permanentemente citados en los textos) y de expresiones o fórmulas similares (estimación común, indigencia, uso) parecen fomentar la idea de que, con alguna diferencia menor, en todos los casos se estaría diciendo lo mismo. En el presente artículo creo haber mostrado que, analizado desde una perspectiva de la filosofía de la economía, esta aparente homogeneidad esconde en verdad interesantes y complejas discrepancias en un tema central para esta disciplina.

Luego de señalar la tensión presente en la obra del Aquinate, este trabajo se centró en la reflexión de tres autores de los siglos XV y XVI. Y lo que los textos muestran es que luego de una referencia explícita a la estimación común como un elemento central para comprender la valoración económica, los caminos de separan. Conrado Summenhart vuelve el peso de la valoración sobre el uso o la utilidad objetiva de las cosas. Domingo de Soto, en cambio, prefiere remitir la valoración a la indigencia que tenemos de los bienes, una indigencia, sin embargo, que no es de la persona particular sino de las personas de una comunidad, y que eventualmente puede ser mejor conocida por un hombre prudente. Diego de Covarrubias, finalmente, se esfuerza por señalar que no hay que ir más allá de la estimación común: es ella la que termina de justificar por qué valoramos de diferente manera, dando a su explicación un giro marcadamente subjetivista. Cualquier apelación al uso o a la necesidad implican para él asociar el valor a la naturaleza del objeto, cosa que a su juicio es evidentemente equivocada. Diferentes comunidades, dice, estiman de diferente manera, e incluso algunas tienen estimaciones vanas, pero, sin embargo, el precio allí también está presente.

Un elemento común que se puede reconocer en todos ellos es el esfuerzo por evitar caer en un objetivismo que consideran absurdo y un subjetivismo que parece difícil de conciliar con el anhelo de un precio justo. Pero la salida 
propuesta por Conrado Summenhart, Domingo de Soto y Diego de Covarrubias son diferentes entre sí, estableciéndose así un debate al que quizás no se ha prestado suficiente atención. Por el contrario, considero que el redescubrimiento de estos contrapuntos permite revalorizar aún más la riqueza de esta tradición intelectual y sus aportes a la filosofía de la economía.

\section{REFERENCIAS BIBLIOGRÁFICAS}

Aristóteles, The Nichomachean Ethics with an english translation by $H$. Rackham. Cambrigde, MA: Harvard University Press, 1994.

Aristóteles, Ética nicomaquea, versión española y notas de A. Gómez Robledo. México: Universidad Nacional Autónoma de México, 1983.

Azevedo Alves, Andre y Moreira, José Manuel, The Salamanca School. New York/London: Continuum, 2010.

Barrientos García, José, Zorroza, María Idoya. "Moral y Política en la Escuela de Salamanca. Presentación", Anuario Filosófico, 45 (2012): 242-245.

Chafuen, Alejandro. Raices cristianas de la economía de Mercado. Santiago: Fundación para el Progreso, 2013

Covarrubias y Leyva, Diego de. Variarum ex iure pontificio, regio, et caesareo resolutionum libri III. Lugduni: Sebastianum Bartolomaei Honorati, 1557. de Roover, Raymond. "Scholastic Economics: Survival and Lasting Influence from the Sixteenth Century to Adam Smith". The Quaterly Journal of Economics. LXIX, n. 2 (1955): 161-190.

del Vigo Gutiérrez, A. "La teoría del justo precio corriente en los moralistas españoles del Siglo de Oro". Burgense: Collectanae Scientifica 20, 1 (1979): 57-130

Dempsey, Bernard W. "Just Price in a Functional Economy". The American Economic Review, 25 (1935): 471-486.

Garrán Martínez, José María. "La justicia en el ámbito mercantil según Domingo de Soto". Ciencia Tomista CXXXII (2005): 111-144.

Gómez Camacho, Francisco. Economía y Filosofía Moral: la formación del pensamiento económico europeo en la Escolástica Española. Madrid: Síntesis, 1998.

Gómez Rivas, León "La teoría del valor en la Escuela de Salamanca". En Modernidad de España, editado por Antonio Miguel Bernal Rodríguez. Madrid: Marcial Pons, 2017.

Gordon, Barry. Economics analysis before Adam Smith. Londres: Macmillan Press, 1975. 
Grice-Hutchinson, Marjorie. The School of Salamanca. Readings in Spanish Monetary Theory 1544-1605. Oxford: Clarendon Press, 1952.

Kaye, Joel. Economy and Nature in the Fourteenth Century. Cambridge: Cambridge University Press, 2004.

Langholm, Odd. The Legacy of Scholasticism in Economic Though. Cambridge: Cambridge University Press, 2006.

Pierre de Jean Olivi. Tratado de los Contratos (Estudio preliminar de R. Ramís Barceló y traducción de P. Ramís Serra y R. Ramís Barcelo). Madrid: Dykinson, 2017.

Popescu, Oreste. Aspectos analíticos en la doctrina del justo Precio en Juan de Matienzo (1520-1579). Buenos Aires: Programa BIBLEH-UCACONICET, 1982.

San Agustín. La Ciudad de Dios (1). Madrid: BAC, 2007.

Schumpeter, Joseph Alois. Historia del Análisis Económico. Barcelona: Ariel, 1971.

Soto, Domingo de. De Iustitia et Iure. Libri Decem/De la justicia y el derecho en diez libros, edición facsimilar de la edición de 1556, traducida por Marcelino González Ordóñez O.P., Vol. III. Madrid: Instituto de Estudios Políticos, 1968.

Summenhart, Conradus. De contractibus licitis atque illicitis. Venecia: Bernardum Iuntam, 1580.

Tomás de Aquino. In decem libros Ethicorum Aristotelis ad Nicomachum expositio. Torino: Marietti, 1964.

Tomás de Aquino. Summa theologiae. Torino Roma, Marietti, 1950.

Alvaro Perpere Viñuales Departamento de Economía Pontificia Universidad Católica Argentina Alicia Moreau de Justo 1400, Edifício Santo Tomás Moro, 4to piso (C1107AFD), Ciudad de Buenos Aires (Argentina) https://orcid.org/0000-0002-3770-7443 
